\title{
Reprodutibilidade e concordância entre diferentes protocolos de baropodometria dinâmica durante a marcha: um estudo preliminar
}

Reproducibility and agreement between different protocols of dynamic baropodometry during gait: a preliminary study

\author{
Reproducibilidad y concordancia entre diferentes protocolos de baropodometría dinámica \\ durante la marcha: un estudio preliminar
}

Fernando Raphael Pinto Guedes Rogerio', Dartagnan Pinto Guedes²

RESUMO | A identificação da pressão plantar durante a marcha é utilizada por clínicos e investigadores para verificar sobrecarga na estrutura podal. Neste particular, diferentes protocolos têm sido empregados para essa finalidade. O objetivo do estudo foi verificar a reprodutibilidade e a concordância de protocolos abreviados de marcha para identificar a pressão plantar dinâmica em jovens assintomáticos. Quinze voluntários foram submetidos aos protocolos abreviados one-step e three-step e ao protocolo padrão midgait em três momentos: inicial, dia seguinte e após sete dias. Para cada uma das oito máscaras podais, foram consideradas as medidas de pico de pressão plantar e integral pressão/ tempo. A reprodutibilidade foi analisada mediante Anova one-way e coeficiente de correlação intraclasse, enquanto a concordância entre os protocolos foi verificada através de teste $t$ pareado, correlação momento-produto de Pearson e plotagem de Bland-Altman. Os resultados apontaram que o protocolo three-step apresentou mais elevada reprodutibilidade em ambas as medidas de pressão plantar dinâmica. Quanto à concordância entre os protocolos, apesar de os abreviados demonstrarem tendência em subestimar as medidas produzidas pelo protocolo padrão midgait, na maioria das máscaras podais não foram identificadas diferenças estatísticas entre os escores médios. Ainda, por intermédio da técnica de Bland-Altman, constatou-se substancial capacidade de concordância entre as medidas identificadas pelos protocolos one-step, three-step e midgait. Concluindo, os protocolos abreviados devem ser selecionados de acordo com a medida de interesse da pressão plantar e a máscara podal a ser analisada, surgindo evidências de reprodutibilidade e concordância mais favoráveis para o uso do protocolo three-step.

Descritores I Pé; Confiabilidade dos Dados; Análise da Marcha.

ABSTRACT I The identification of plantar pressure during gait is used by clinicians and researchers to verify burden on the plantar structure. In this study, different protocols have been employed for this purpose. The aim of this study was to verify the reproducibility and agreement of shortened gait protocols to identify dynamic plantar pressure in asymptomatic young people. Fifteen volunteers were submitted to the shortened one-step and three-step protocols and to the standard midgait protocol in three moments: initial, after one day and after seven days. For each of the eight foot masks, the measurements of peak plantar pressure and pressure-time integral were considered. Reproducibility was analyzed by one-way Anova test and intraclass correlation coefficient, while the agreement between the protocols was verified through paired t-test, Pearson's moment-product correlation and Bland-Altman plot. The results showed that the three-step protocol presented higher reproducibility in both measurements of dynamic plantar pressure. Regarding the agreement between protocols, although the shortened ones showed a tendency to underestimate 
the standard midgait measurements, most foot masks showed no statistical differences between mean scores. Furthermore, through the Bland-Altman technique, there was substantial agreement capacity between the one-step, three-step and midgait protocols measurements. In conclusion, the shortened protocols should be selected according to the measurement of interest of plantar pressure and foot masks to be analyzed. The evidence of reproducibility and agreement was more favorable for the use of the three-step protocol.

Keywords | Foot; Data Accuracy; Gait Analysis.

RESUMEN | Los médicos e investigadores utilizan la identificación de la presión plantar durante la marcha para verificar la sobrecarga en la estructura podal. Para ello, emplean diferentes protocolos. El objetivo de este estudio fue verificar la reproducibilidad y la concordancia de los protocolos de caminata abreviados para identificar la presión plantar dinámica en jóvenes asintomáticos. Para esto, participaron quince voluntarios en protocolos abreviados one-step y three-step y en el protocolo estándar midgait en tres momentos: inicial, día siguiente y después de siete días. Para cada una de las máscaras podales, se consideraron la medición máxima de la presión plantar e integral presión/tiempo. Para analizar la reproducibilidad, se usó la Anova one-way y el coeficiente de correlación intraclase, mientras que la concordancia entre los protocolos se verificó mediante la prueba t pareada, la correlación del producto-momento de Pearson y el gráfico de Bland-Altman. Los resultados mostraron que el protocolo threestep obtuvo una mayor reproducibilidad en ambas las medidas de presión plantar dinámica. En cuanto a la concordancia entre los protocolos, aunque los protocolos abreviados muestran una tendencia a subestimar las medidas producidas por el protocolo estándar midgait, en la mayoría de las mascarillas podales no se identificaron diferencias estadísticas entre las puntuaciones medias. En la técnica de Bland-Altman, hubo una capacidad sustancial de concordancia entre las medidas identificadas por los protocolos one-step, three-step y midgait. Se concluye que los protocolos abreviados deben seleccionarse según la medida de interés de la presión plantar y la mascarilla podal que se debe analizar, con evidencia de reproducibilidad y concordancia más favorable para el uso del protocolo three-step.

Palabras Clave | Pie; Exactitud de los Datos; Análisis de la Marcha..

\section{INTRODUÇÃO}

A análise da pressão plantar em condições dinâmicas (PPD) é um importante componente no diagnóstico e no tratamento de diversas condições patológicas ${ }^{1}$ e de desempenho físico ${ }^{2}$. A aplicação clínica e industrial da PPD promove maior eficiência no delineamento de órteses esportivas ${ }^{3}$, na prevenção de lesões ${ }^{4}$, no controle e avaliação de ulcerações diabéticas ${ }^{5}$ e, mais recentemente, na terapia de biofeedback ${ }^{6}$.

O monitoramento da PPD é realizado mediante sistema de baropodometria eletrônica que, entre outros procedimentos, utiliza-se de plataforma de pressão posicionada no solo, em que o indivíduo entra em contato com o equipamento em diferentes condições experimentais, inclusive em esteiras e palmilhas posicionadas na interface calçado/pé7.

Medidas relacionadas à PPD da marcha humana solicitam inúmeras coletas a fim de realizar análise representativa do padrão habitual. Nesse sentido, diferentes protocolos têm sido propostos com intuito de promover análises mais acessíveis quanto à duração dos procedimentos, à fadiga do paciente, ao espaço físico e à confiabilidade na identificação dos indicadores da PPD em atividades dinâmicas ${ }^{8}$.
Dentre os recursos disponíveis para análise da marcha, o protocolo midgait é considerado padrão ouro para identificar as medidas de PPD. Neste particular, o protocolo midgait preconiza que o indivíduo descalço percorra em uma mesma direção a distância próxima de 10 metros, em velocidade de marcha estável e auto selecionada, com o baropodômetro posicionado no meio do percurso9. Por vezes, devido à distância a ser percorrida, à dificuldade de familiarização aos seus procedimentos e ao tempo de coleta dos dados, esse protocolo tornar-se limitado para uso em ambiente clínico e em indivíduos com processos dolorosos, risco de quedas e dificuldade de locomoção.

Desse modo, com intenção de facilitar as análises da PPD, protocolos abreviados de marcha têm sido sugeridos a fim de minimizar dificuldades relacionadas ao uso do protocolo midgait ${ }^{10-12}$, tornando os procedimentos clinicamente mais factíveis. Dentre os protocolos abreviados preconizados, destacam-se os de inicialização da marcha denominados one-step e three-step, em que o indivíduo entra em contato com o baropodômetro no primeiro ou no terceiro passo inicial da marcha, respectivamente.

Embora os protocolos abreviados sejam de menor complexidade - diferentemente do protocolo midgait -, eles impõem diferentes demandas na habilidade de 
locomoção, principalmente em razão da análise ser realizada nos primeiros passos da marcha. Assim, os protocolos abreviados exercem diferentes demandas sobre o sistema de controle postural quanto à aceleração, ao equilíbrio e à perturbação externa, desencadeando, portanto, disparidades nos padrões de movimento durante a marcha. Sendo assim, torna-se possível assumir a hipótese de que os protocolos abreviados de marcha podem gerar resultados conflitantes no monitoramento da PPD.

Estudos prévios investigaram a concordância e a reprodutibilidade de diferentes protocolos de análise da PPD na marcha de jovens e adultos em diferentes condições experimentais ${ }^{13-15}$. Apesar dos estudos demonstrarem boa concordância entre os métodos, eles não investigaram ambos os protocolos abreviados, simultaneamente, frente ao padrão ouro, gerando dúvida sobre o protocolo abreviado mais indicado para o uso. Os objetivos do presente estudo foram identificar: (1) a reprodutibilidade das medidas de PPD registradas em três momentos distintos (inicial, após um dia e após sete dias) mediante o uso dos protocolos one-step e three-step e (2) a concordância entre as medidas de PPD registradas mediante o uso dos protocolos midgait, one-step e three-step em jovens assintomáticos.

\section{METODOLOGIA}

Os voluntários foram informados sobre os procedimentos metodológicos e confirmaram a participação no estudo mediante termo de consentimento livre e esclarecido.

\section{Participantes}

Recorreu-se a uma amostra não probabilística composta por 15 universitários de ambos os sexos, assintomáticos, com idade entre 18 e 30 anos. Foram considerados critérios de exclusão para participar do estudo: lesão em algum segmento dos membros inferiores nos últimos seis meses, fratura óssea recente, procedimentos cirúrgicos prévios, excesso de peso corporal, diagnóstico de patologias ortopédicas, neurológicas, cardiorrespiratórios ou vestibulares. Os participantes preencheram um questionário estruturado com finalidade de reunir dados demográficos e informações autorrelatadas sobre o estado de saúde atual. Foram realizadas, ainda, medidas de peso corporal e de estatura para cálculo do índice de massa corporal (quociente entre a medida de peso corporal em quilogramas e estatura em metros elevada ao quadrado $-\mathrm{kg} / \mathrm{m}^{2}$ ).

\section{Equipamento}

Para coleta dos dados referente as medidas de PPD nos diferentes protocolos de marcha, foi utilizada plataforma de pressão modelo Footwork Pro AM3 - França, com superfície ativa de 490×490mm, espessura de 4mm, 4096 captadores capacitivos calibrados, captor de 7,62×7,62mm de medida, frequência de $200 \mathrm{~Hz}$ e pressão máxima por captor de $120 \mathrm{~N} / \mathrm{cm}^{2}$.

\section{Procedimento experimental}

Inicialmente foi demonstrado aos participantes os três protocolos utilizados para análise das medidas de PPD e, na sequência, os participantes foram submetidos ao processo de familiarização com seus procedimentos. A familiarização consistiu da execução de pelo menos três repetições de cada protocolo; contudo, nos casos em que o participante demonstrou não ter compreendido plenamente os procedimentos ou apresentou dificuldade na execução do protocolo, foram realizadas repetições adicionais. Posteriormente, foram realizadas demarcações no solo separadamente para cada protocolo com o intuito de definir a posição de partida adequada para uma execução bem-sucedida. Em seguida, foi realizada a calibração do equipamento e inseridas, no sistema eletrônico, as informações do participante necessárias para atendimento dos protocolos de medida.

Todos os participantes foram submetidos aos três diferentes protocolos de marcha: midgait, one-step e threestep. A sequência de aplicação dos protocolos foi sorteada aleatoriamente. Os participantes caminhavam naturalmente em velocidade auto-selecionada, com os braços ao longo do corpo, olhar no plano horizontal, se deslocando um, dois ou cinco metros previamente ao contato com a plataforma de pressão e continuavam caminhando mesma distância após o contato inicial ${ }^{9,10,12}$. Foram realizadas seis repetições dos movimentos de caminhada em cada protocolo, tendo como referência para análise da PPD somente as respostas equivalentes ao pé esquerdo, conforme preconizado por Menz et al. ${ }^{16}$. Nos casos em que os participantes não apresentavam dados que atendessem os critérios de aceitação, os procedimentos foram repetidos de maneira subsequente até atingir a quantidade necessária de pisadas. $\mathrm{O}$ procedimento foi repetido em condições similares após um e sete dias.

Os critérios de rejeição dos dados coletados foram: tentativa em que o pé não contatou totalmente com a plataforma; anormalidades intencionais na marcha observada pelo investigador; alteração no ritmo da marcha para adequar os passos previamente ao contato com a 
plataforma; desequilíbrio durante a marcha; início da marcha fora do espaço demarcado ou a não conclusão da sequência de passos após o contato com o equipamento.

\section{Medidas analisadas pela baropodometria dinâmica}

As medidas de PPD foram identificadas por intermédio dos diferentes protocolos em três momentos distintos: inicial, após um dia e sete dias. Optou-se por aplicar os protocolos em três momentos diferentes na tentativa de garantir consistência das características da marcha dos participantes. Nesse sentido, foram reunidas medidas equivalentes ao pico de pressão plantar (PPP) e à integral pressão/tempo (IPT). Ambas as medidas foram selecionadas devido a sua aplicação clínica nas disfunções associadas aos pés, risco de ulcerações plantares e efetividade nas intervenções terapêuticas ${ }^{17,18}$.

O PPP e a IPT foram dimensionados por meio da função AutoMask do software FootWork Pro 2.9.1. Foram consideradas oito máscaras podais: hálux, outros dedos, primeiro metatarso, segundo e terceiro metatarso, quarto e quinto metatarso, médio pé, região interna de calcâneo e região externa de calcâneo. Essas regiões foram selecionadas para fornecer informações detalhadas sobre a função de diferentes áreas plantares durante a marcha ${ }^{14,15}$.

\section{Análise estatística}

A análise estatística dos dados foi realizada mediante o software Statistical Package for the Social Science, versão 22 (SPSS, Inc., Chicago, IL, EUA). Previamente às análises inferenciais, foi comprovada a distribuição normal dos dados referentes ao PPP e à IPT por intermédio do teste de Shapiro-Wilk; logo, recorreu-se aos procedimentos da estatística descritiva paramétrica (média \pm desvio-padrão). Escores representativos do PPP e da IPT em cada protocolo foram estabelecidos mediante o cálculo de média das seis execuções. A reprodutibilidade dos dados envolvendo os três momentos (inicial, após um e sete dias) obtidos por intermédio dos diferentes protocolos foi identificada mediante Anova One-way e coeficiente de correlação intraclasse $(\mathrm{CCI})$. A concordância entre escores de PPP e IPT mediante o uso dos protocolos midgait, one-step e three-step foi analisada mediante teste $t$ de Student para dados pareados, correlação momento-produto de Pearson, diferença média das variações individuais acompanhada do respectivo desvio-padrão e plotagem de Bland-Altman.

\section{RESULTADOS}

\section{Características da amostra}

A amostra foi constituída por oito mulheres e sete homens com idade média equivalente a $23,68 \pm 2,80$ anos, peso corporal $64,52 \pm 10,55 \mathrm{~kg}$, estatura $168,68 \pm 7,93 \mathrm{~cm}$ e índice de massa corporal $22,87 \pm 3,14 \mathrm{~kg} / \mathrm{m}^{2}$.

\section{Reprodutibilidade do PPP e da IPT}

As informações estatísticas referentes à reprodutibilidade das medidas de PPP e IPT são disponibilizadas na Tabela 1. Em ambos os casos, os valores de $\mathrm{F}$ encontrados não apontaram diferenças significativas estatisticamente entre os três momentos de coleta dos dados. As dimensões do CCI sugerem que o protocolo three-step apresentou dados de PPP e IPT com mais elevada reprodutibilidade. Os dados das máscaras podais referentes ao segundo/terceiro metatarsos e ao interno de calcâneo foram os que revelaram mais aprimorada reprodutibilidade, enquanto os dados das máscaras podais referentes a outros dedos e ao médio pé foram os que demonstraram reprodutibilidade menos aprimorada, nomeadamente mediante o uso do protocolo one-step.

Tabela 1. Reprodutibilidade de medidas equivalentes ao pico de pressão plantar e à integral pressão/tempo encontrada em diferentes momentos mediante os protocolos abreviados one-step e three-step

\begin{tabular}{|c|c|c|c|c|c|}
\hline \multirow{2}{*}{$\begin{array}{l}\text { Máscaras } \\
\text { podais }\end{array}$} & & \multicolumn{2}{|c|}{ Pico de pressão plantar ( $\mathrm{KPa})$} & \multicolumn{2}{|c|}{ Integral pressão/tempo $(\mathrm{KPa} / \mathrm{s})$} \\
\hline & & One-step & Three-step & One-step & Three-step \\
\hline Hálux & $\begin{array}{c}M_{0} \\
M_{1} \\
M_{7} \\
\text { Teste F } \\
\text { CCl }\end{array}$ & $\begin{array}{c}368,61 \pm 83,72 \\
316,31 \pm 93,74 \\
325,01 \pm 122,28 \\
2,498(\mathrm{~ns}) \\
0,78\end{array}$ & $\begin{array}{c}357,36 \pm 95,03 \\
328,11 \pm 99,77 \\
340,35 \pm 92,59 \\
0,817 \text { (ns) } \\
0,79\end{array}$ & $\begin{array}{c}21,11 \pm 8,27 \\
20,60 \pm 9,35 \\
20,24 \pm 13,09 \\
0,020 \text { (ns) } \\
0,96\end{array}$ & $\begin{array}{c}23,57 \pm 10,53 \\
22,78 \pm 11,16 \\
22,67 \pm 9,78 \\
0,098 \text { (ns) } \\
0,85\end{array}$ \\
\hline Outros dedos & $\begin{array}{c}M_{0} \\
M_{1} \\
M_{7} \\
\text { Teste } \mathrm{F} \\
\mathrm{CCl}\end{array}$ & $\begin{array}{c}289,75 \pm 122,32 \\
295,36 \pm 75,97 \\
300,84 \pm 78,13 \\
0,065 \text { (ns) } \\
0,44\end{array}$ & $\begin{array}{c}288,86 \pm 134,31 \\
249,04 \pm 147,36 \\
268,62 \pm 121,88 \\
0,734(\mathrm{~ns}) \\
0,79\end{array}$ & $\begin{array}{c}11,55 \pm 9,27 \\
13,45 \pm 12,66 \\
13,23 \pm 9,96 \\
0,483 \text { (ns) } \\
0,63\end{array}$ & $\begin{array}{c}11,92 \pm 7,37 \\
12,03 \pm 10,03 \\
16,07 \pm 10,88 \\
2,202 \text { (ns) } \\
0,78\end{array}$ \\
\hline
\end{tabular}


Tabela 1. Continuação

\begin{tabular}{|c|c|c|c|c|c|}
\hline \multirow{2}{*}{$\begin{array}{l}\text { Máscaras } \\
\text { podais }\end{array}$} & & \multicolumn{2}{|c|}{ Pico de pressão plantar ( $\mathrm{KPa})$} & \multicolumn{2}{|c|}{ Integral pressão/tempo (KPa/s) } \\
\hline & & One-step & Three-step & One-step & Three-step \\
\hline $1^{\circ}$ metatarso & $\begin{array}{c}M_{0} \\
M_{1} \\
M_{7} \\
\text { Teste F } \\
\text { CCl }\end{array}$ & $\begin{array}{c}295,65 \pm 81,77 \\
268,36 \pm 54,85 \\
272,48 \pm 60,92 \\
1,019 \text { (ns) } \\
0,54\end{array}$ & $\begin{array}{c}311,79 \pm 54,93 \\
279,48 \pm 48,65 \\
288,61 \pm 51,06 \\
2,048 \text { (ns) } \\
0,80\end{array}$ & $\begin{array}{c}29,67 \pm 11,92 \\
30,43 \pm 13,81 \\
27,94 \pm 13,40 \\
0,190 \text { (ns) } \\
0,50\end{array}$ & $\begin{array}{c}34,68 \pm 10,87 \\
38,95 \pm 14,03 \\
40,25 \pm 12,45 \\
2,993 \text { (ns) } \\
0,88\end{array}$ \\
\hline $\begin{array}{l}2^{\circ} \text { e } 3^{\circ} \\
\text { metatarsos }\end{array}$ & $\begin{array}{c}M_{0} \\
M_{1} \\
M_{7} \\
\text { Teste F } \\
\text { CCl }\end{array}$ & $\begin{array}{c}388,72 \pm 52,55 \\
398,33 \pm 52,20 \\
396,20 \pm 47,88 \\
1,014 \text { (ns) } \\
0,86\end{array}$ & $\begin{array}{c}402,59 \pm 38,87 \\
394,33 \pm 35,37 \\
405,13 \pm 54,83 \\
0,757 \text { (ns) } \\
0,94\end{array}$ & $\begin{array}{c}50,42 \pm 9,89 \\
52,72 \pm 9,38 \\
52,42 \pm 9,08 \\
1,619 \text { (ns) } \\
0,84\end{array}$ & $\begin{array}{c}54,99 \pm 6,19 \\
56,89 \pm 4,44 \\
57,62 \pm 6,09 \\
2,444 \text { (ns) } \\
0,87\end{array}$ \\
\hline $\begin{array}{l}4^{\circ} \text { e } 5^{\circ} \\
\text { metatarsos }\end{array}$ & $\begin{array}{c}M_{0} \\
M_{1} \\
M_{7} \\
\text { Teste F } \\
\text { CCl }\end{array}$ & $\begin{array}{c}371,93 \pm 86,34 \\
389,68 \pm 74,19 \\
377,54 \pm 66,53 \\
0,593 \text { (ns) } \\
0,84\end{array}$ & $\begin{array}{c}373,51 \pm 63,47 \\
376,60 \pm 64,76 \\
356,12 \pm 73,07 \\
1,022 \text { (ns) } \\
0,82\end{array}$ & $\begin{array}{c}30,35 \pm 13,04 \\
36,83 \pm 12,64 \\
32,83 \pm 9,61 \\
2,600 \text { (ns) } \\
0,79\end{array}$ & $\begin{array}{c}35,58 \pm 10,74 \\
34,70 \pm 10,81 \\
34,36 \pm 9,57 \\
0,306 \text { (ns) } \\
0,93\end{array}$ \\
\hline Médio pé & $\begin{array}{c}M_{0} \\
M_{1} \\
M_{7} \\
\text { Teste F } \\
\text { CCl }\end{array}$ & $\begin{array}{c}70,57 \pm 51,95 \\
102,71 \pm 67,73 \\
97,37 \pm 63,55 \\
1,442(\mathrm{~ns}) \\
0,40\end{array}$ & $\begin{array}{c}61,15 \pm 39,44 \\
56,43 \pm 40,90 \\
53,47 \pm 42,42 \\
0,391 \text { (ns) } \\
0,74\end{array}$ & $\begin{array}{c}4,82 \pm 3,85 \\
6,70 \pm 5,04 \\
6,67 \pm 6,39 \\
0,485 \text { (ns) } \\
0,55\end{array}$ & $\begin{array}{c}3,73 \pm 2,42 \\
4,59 \pm 2,88 \\
3,94 \pm 3,07 \\
1,737 \text { (ns) } \\
0,81\end{array}$ \\
\hline $\begin{array}{l}\text { Interno de } \\
\text { calcâneo }\end{array}$ & $\begin{array}{c}M_{0} \\
M_{1} \\
M_{7} \\
\text { Teste F } \\
\text { CCl }\end{array}$ & $\begin{array}{c}373,73 \pm 37,80 \\
377,83 \pm 47,73 \\
390,38 \pm 41,52 \\
2,294 \text { (ns) } \\
0,82\end{array}$ & $\begin{array}{c}393,43 \pm 55,47 \\
404,18 \pm 37,65 \\
403,13 \pm 46,41 \\
0,606(n s) \\
0,87\end{array}$ & $\begin{array}{c}40,20 \pm 10,35 \\
40,56 \pm 10,22 \\
40,26 \pm 10,21 \\
0,009 \text { (ns) } \\
0,93\end{array}$ & $\begin{array}{c}32,89 \pm 7,94 \\
33,84 \pm 8,41 \\
33,72 \pm 9,75 \\
0,125 \text { (ns) } \\
0,90\end{array}$ \\
\hline $\begin{array}{l}\text { Externo de } \\
\text { calcâneo }\end{array}$ & $\begin{array}{c}M_{0} \\
M_{1} \\
M_{7} \\
\text { Teste F } \\
\text { CCl }\end{array}$ & $\begin{array}{c}375,32 \pm 44,43 \\
365,98 \pm 43,32 \\
381,76 \pm 46,76 \\
1,153 \text { (ns) } \\
0,81\end{array}$ & $\begin{array}{c}402,49 \pm 63,29 \\
420,62 \pm 47,20 \\
412,20 \pm 50,56 \\
1,282 \text { (ns) } \\
0,86\end{array}$ & $\begin{array}{c}32,46 \pm 9,45 \\
31,64 \pm 7,08 \\
33,58 \pm 7,51 \\
0,383 \text { (ns) } \\
0,70\end{array}$ & $\begin{array}{c}28,15 \pm 7,31 \\
28,01 \pm 6,80 \\
27,74 \pm 7,57 \\
0,029 \text { (ns) } \\
0,80\end{array}$ \\
\hline
\end{tabular}

$M_{0}$ : Momento inicial; $M_{1}$ : Após um dia; $M_{7}$ : Após 7 dias.

\section{Concordância dos dados equivalentes ao PPP e à IPT}

Não sendo identificadas diferenças estatísticas entre as medidas realizadas no momento inicial após um e sete dias, recorreu-se aos escores da medida inicial como representativos para análise de concordância entre ambos os protocolos abreviados e o protocolo padrão midgait. Informações estatísticas da concordância entre os dados equivalentes ao PPP produzidos pelos três protocolos são mostrados na Tabela 2.

$\mathrm{Na}$ comparação com o protocolo midgait, enquanto o uso do protocolo one-step apontou diferenças significativas nas máscaras podais correspondes às regiões de calcâneo interno $(t=3,411 ; p=0,01)$ e externo $(t=2,393 ; p=0,03)$, o protocolo three-step apresentou diferença estatística apenas na máscara podal correspondente ao primeiro metatarso $(t=4,793$; $\mathrm{p}<0,01)$. As demais máscaras podais não apresentaram diferenças estatísticas, o que, associados aos significativos valores de $\mathrm{r}$ e as menores dimensões equivalentes às diferenças médias das variações individuais, apontam expressiva concordância entre o protocolo midgait e ambos os protocolos abreviados.

Informações estatísticas associadas à concordância entre os dados equivalentes ao IPT produzidos pelos três protocolos são disponibilizados na Tabela 3. No caso do protocolo one-step, foi encontrada diferença significativa unicamente na comparação com o protocolo padrão midgait, nas medidas da máscara podal corresponde à região do primeiro metatarso $(t=2,456 ; \mathrm{p}=0,03)$; ao passo que, ao estabelecer comparações entre os protocolos three-step e midgait, a diferença significativa foi verificada nas medidas correspondentes ao quarto/quinto metatarsos $(t=2,615$; $\mathrm{p}=0,02)$. As demais máscaras podais não apresentaram diferenças estatísticas e registraram valores de $\mathrm{r}$ significativos estatisticamente. As diferenças médias das variações individuais entre as medidas produzidas pelo uso dos três protocolos sugerem maior concordância entre o protocolo padrão midgait e o protocolo abreviado three-step. 
Tabela 2. Concordância entre medidas equivalentes ao pico de pressão plantar (KPa) encontrada mediante o uso dos protocolos midgait, one-step e three-step

\begin{tabular}{|c|c|c|c|c|c|c|}
\hline \multirow[b]{2}{*}{ Máscaras podais } & \multirow[b]{2}{*}{ Protocolo } & \multirow[b]{2}{*}{$M \pm D P$} & \multirow[b]{2}{*}{ Teste $\mathrm{t}$} & \multirow[b]{2}{*}{$\mathbf{r}$} & \multicolumn{2}{|c|}{ Plotagem Bland-Altman } \\
\hline & & & & & $\begin{array}{l}\text { Diferença } \\
\text { média }\end{array}$ & $\begin{array}{l}\text { Limites de concordância } \\
\text { ( } \pm 2 \text { DP) }\end{array}$ \\
\hline Hálux & $\begin{array}{l}\text { Midgait } \\
\text { One-step } \\
\text { Three-step }\end{array}$ & $\begin{array}{c}389,72 \pm 90,95 \\
368,61 \pm 83,72 \\
357,36 \pm 95,03\end{array}$ & $\begin{array}{l}1,138 \text { (ns) } \\
1,588 \text { (ns) }\end{array}$ & $\begin{array}{l}0,58(p=0,02) \\
0,59(p=0,02)\end{array}$ & $\begin{array}{l}-25,9 \\
-37,1\end{array}$ & $\begin{array}{l}-198,4-146,6 \\
-214,6-140,4\end{array}$ \\
\hline Outros dedos & $\begin{array}{l}\text { Midgait } \\
\text { One-step } \\
\text { Three-step }\end{array}$ & $\begin{array}{c}281,05 \pm 97,93 \\
289,75 \pm 122,32 \\
288,86 \pm 134,31\end{array}$ & $\begin{array}{l}0,328 \text { (ns) } \\
0,232 \text { (ns) }\end{array}$ & $\begin{array}{l}0,58(p=0,02) \\
0,46(p=0,04)\end{array}$ & $\begin{array}{l}8,7 \\
19,8\end{array}$ & $\begin{array}{c}-192,4-209,8 \\
-193,6-233,1\end{array}$ \\
\hline 1- metatarso & $\begin{array}{c}\text { Midgait } \\
\text { One-step } \\
\text { Three-step }\end{array}$ & $\begin{array}{l}326,62 \pm 33,23 \\
295,65 \pm 81,77 \\
275,98 \pm 46,86\end{array}$ & $\begin{array}{c}1,442(n s) \\
4,793(p<0,01)\end{array}$ & $\begin{array}{l}0,41(p=0,05) \\
0,52(p=0,05)\end{array}$ & $\begin{array}{l}-31,0 \\
-50,6\end{array}$ & $\begin{array}{l}-194,0-132,1 \\
-130,9-29,6\end{array}$ \\
\hline $\begin{array}{l}2^{\circ} \text { e } 3^{\circ} \\
\text { metatarsos }\end{array}$ & $\begin{array}{l}\text { Midgait } \\
\text { One-step } \\
\text { Three-step }\end{array}$ & $\begin{array}{l}408,11 \pm 56,65 \\
388,72 \pm 52,55 \\
402,59 \pm 38,87\end{array}$ & $\begin{array}{l}1,684 \text { (ns) } \\
0,603 \text { (ns) }\end{array}$ & $\begin{array}{l}0,67(p=0,01) \\
0,78(p<0,01)\end{array}$ & $\begin{array}{l}-19,4 \\
-5,5\end{array}$ & $\begin{array}{l}-106,8-68,0 \\
-74,9-63,9\end{array}$ \\
\hline $\begin{array}{l}4^{\circ} \text { e } 5^{\circ} \\
\text { metatarsos }\end{array}$ & $\begin{array}{l}\text { Midgait } \\
\text { One-step } \\
\text { Three-step }\end{array}$ & $\begin{array}{l}351,20 \pm 34,42 \\
371,93 \pm 86,34 \\
373,51 \pm 63,47\end{array}$ & $\begin{array}{l}0,856 \text { (ns) } \\
1,909 \text { (ns) }\end{array}$ & $\begin{array}{l}0,39(p=0,05) \\
0,72(p=0,01)\end{array}$ & $\begin{array}{l}20,7 \\
22,3\end{array}$ & $\begin{array}{c}-163,1-204,5 \\
-66,4-111,0\end{array}$ \\
\hline Médio pé & $\begin{array}{l}\text { Midgait } \\
\text { One-step } \\
\text { Three-step }\end{array}$ & $\begin{array}{l}67,21 \pm 37,57 \\
70,57 \pm 51,95 \\
61,15 \pm 39,44\end{array}$ & $\begin{array}{l}0,253 \text { (ns) } \\
0,541 \text { (ns) }\end{array}$ & $\begin{array}{l}0,46(p=0,04) \\
0,44(p=0,05)\end{array}$ & $\begin{array}{l}3,4 \\
-6,1\end{array}$ & $\begin{array}{l}-97,6-104,3 \\
-91,0-78,9\end{array}$ \\
\hline $\begin{array}{l}\text { Interno de } \\
\text { calcâneo }\end{array}$ & $\begin{array}{l}\text { Midgait } \\
\text { One-step } \\
\text { Three-step }\end{array}$ & $\begin{array}{c}411,53 \pm 37,27 \\
373,73 \pm 37,80 \\
393,43 \pm 55,47\end{array}$ & $\begin{array}{c}3,411(p=0,01) \\
1,957(n s)\end{array}$ & $\begin{array}{c}0,34 \text { (ns) } \\
0,82(p<0,01)\end{array}$ & $\begin{array}{l}-37,8 \\
-18,1\end{array}$ & $\begin{array}{l}-121,9-46,3 \\
-81,7-45,5\end{array}$ \\
\hline $\begin{array}{l}\text { Externo de } \\
\text { calcâneo }\end{array}$ & $\begin{array}{c}\text { Midgait } \\
\text { One-step } \\
\text { Three-step }\end{array}$ & $\begin{array}{l}408,28 \pm 53,69 \\
375,32 \pm 44,43 \\
402,49 \pm 63,29\end{array}$ & $\begin{array}{c}2,393(p=0,03) \\
0,535(n s)\end{array}$ & $\begin{array}{c}0,41(n s) \\
0,75(p=0,01)\end{array}$ & $\begin{array}{l}-33,0 \\
-5,8\end{array}$ & $\begin{array}{l}-137,5-71,6 \\
-87,8-76,2\end{array}$ \\
\hline
\end{tabular}

Tabela 3. Concordância entre medidas equivalentes à integral pressão/tempo (KPa/s) encontrada mediante o uso dos protocolos midgait, one-step e three-step

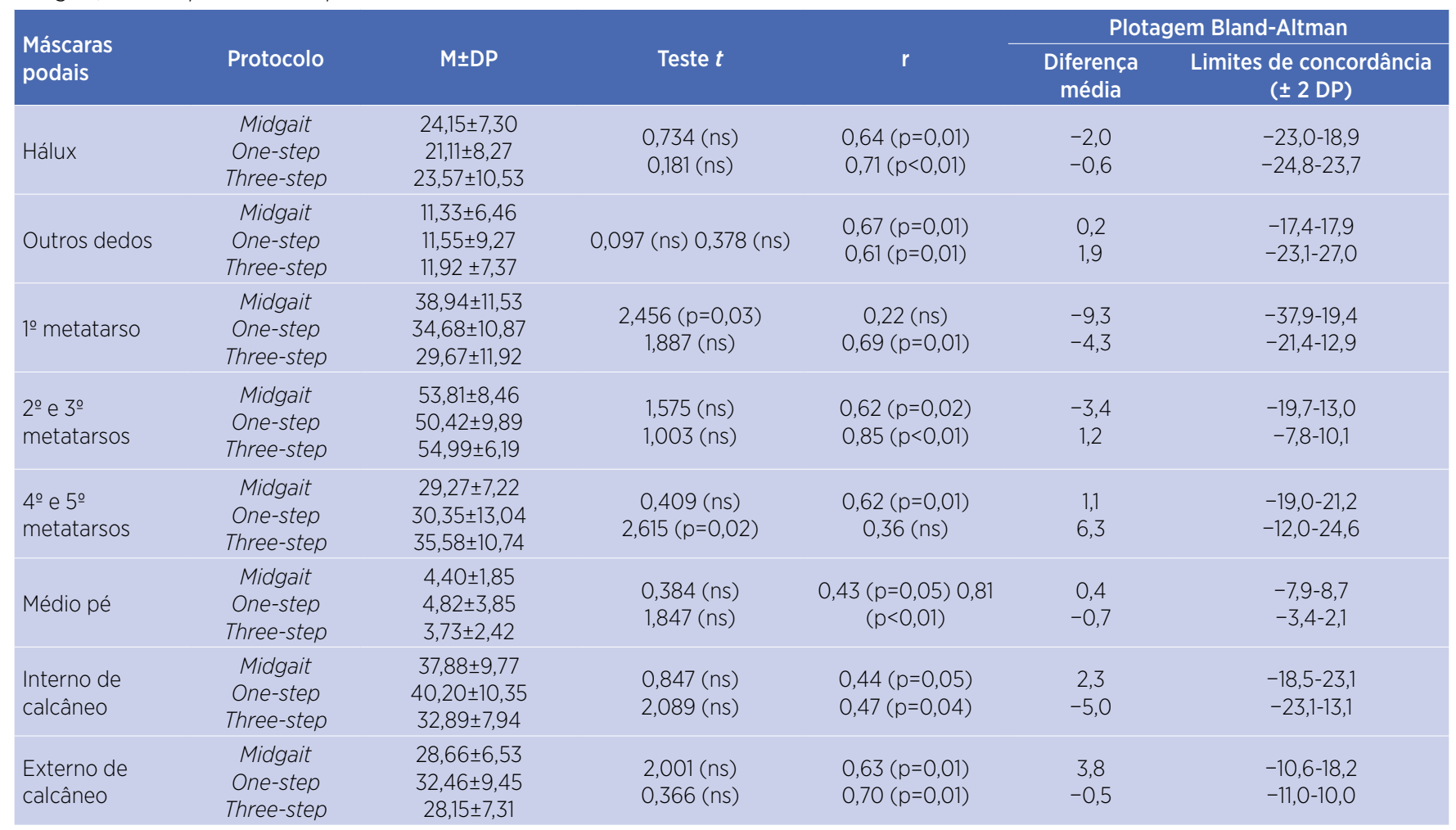


Nas Figuras 1 e 2 são ilustrados os diagramas de dispersão com plotagem da média dos dados identificados por cada um dos protocolos abreviados (one-step e three-step) e pelo protocolo midgait (abscissa), além das diferenças entre os dados individuais identificados pelos três protocolos (ordenada). O procedimento de análise proposto por BlandAltman permite visualizar as diferenças individuais e os limites extremos de concordância ( \pm 2 desvios-padrão da diferença) observados na comparação entre cada protocolo abreviado e o protocolo midgait. A disposição gráfica dos dados sugere menor concordância entre os protocolos midgait e one-step. Quando da comparação entre os dados do PPP e da IPT, produzidos por cada um dos protocolos abreviados e pelo protocolo midgait, são encontradas diferenças médias próximas de zero e limites de concordância razoavelmente baixos nas oito máscaras podais - sobretudo no caso do PPP e do protocolo three-step.
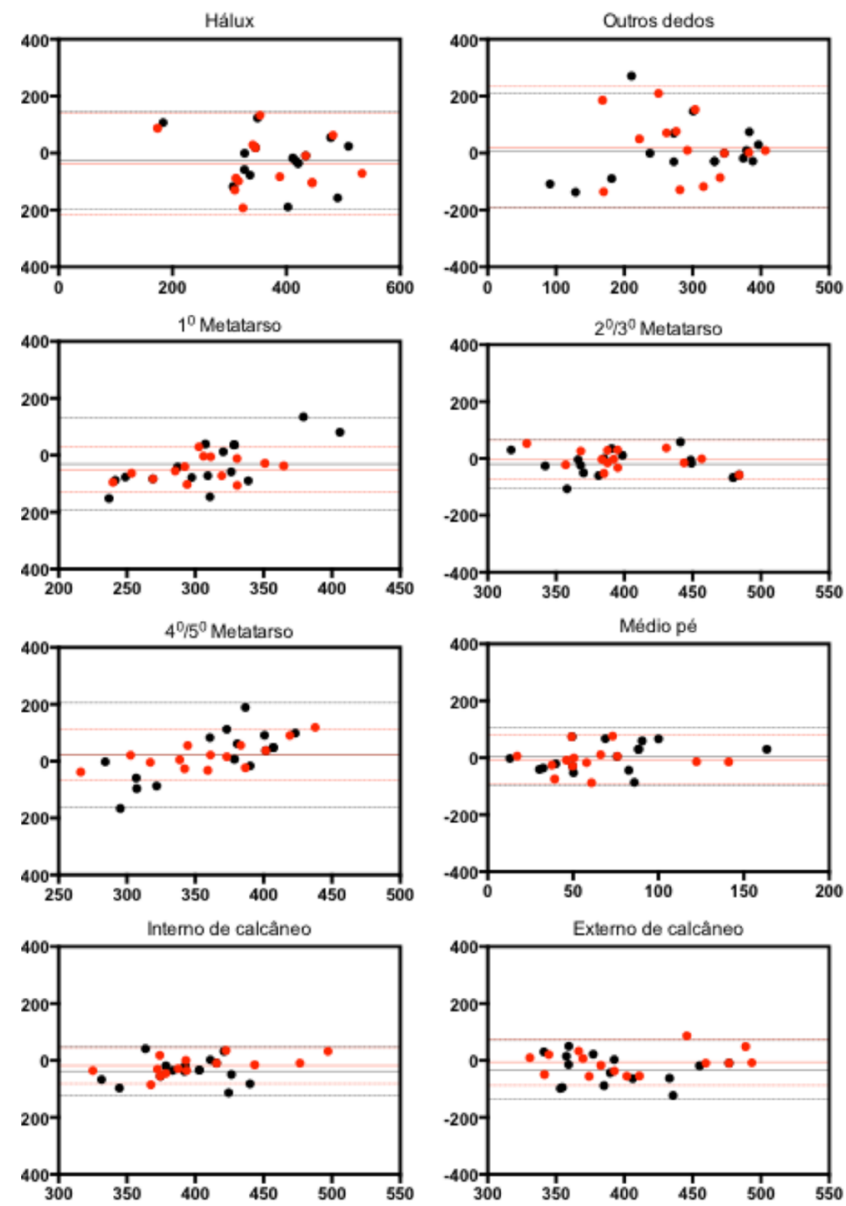

Ordenada: diferenças entre os valores identificados pelos protocolos midgait, one-step e three-step.

Abscissa: média dos valores identificados pelos protocolos midgait, one-step e three-step.

Figura 1. Plotagem de Bland-Altman para os limites de concordância entre medidas de pico de pressão plantar (Kpa) identificados pelos protocolos midgait, one-step $(\bullet)$ e three-step $(\bullet)$.
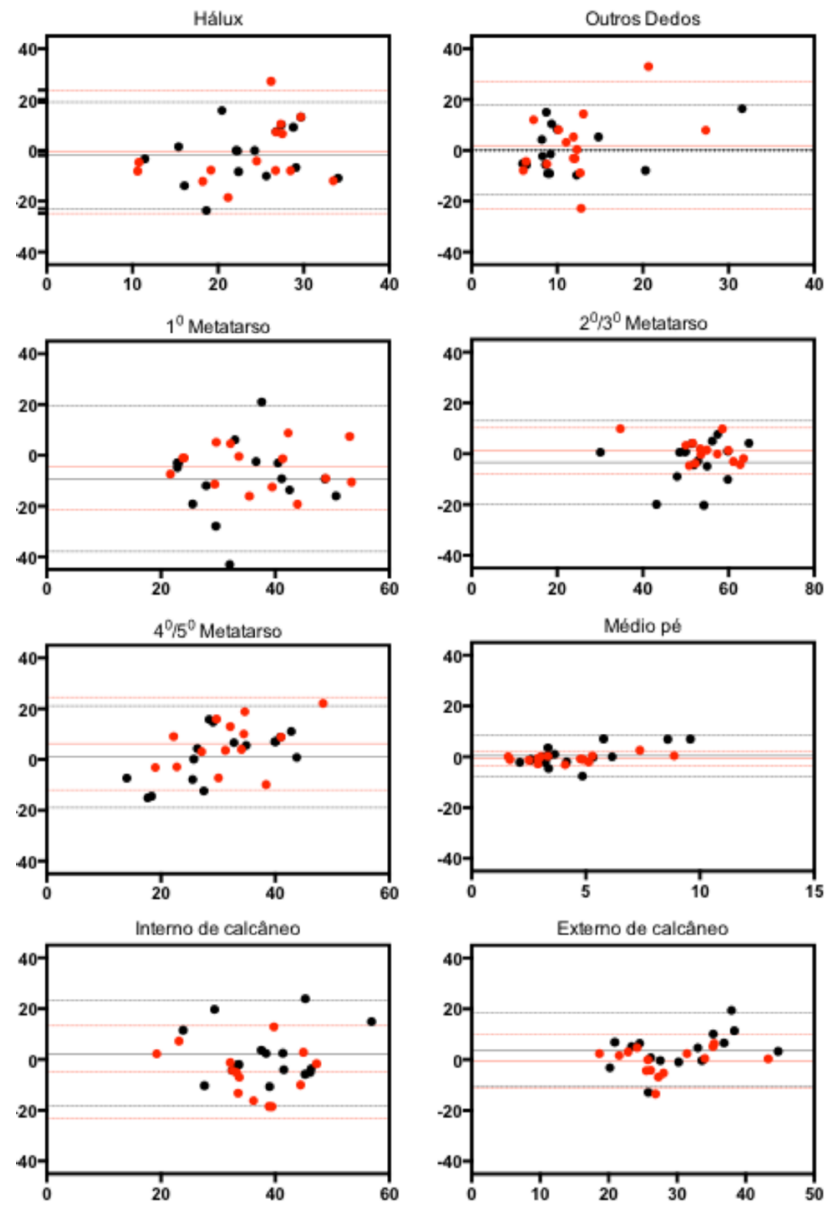

Ordenada: diferencas entre os valores identificados pelos protocolos midgait, one-step e three-step.

Abscissa: média dos valores identificados pelos protocolos midgait, one-step e three-step.

Figura 2. Plotagem de Bland-Altman para os limites de concordância entre medidas de integral pressão/tempo (Kpa/s.) identificados pelos protocolos midgait, one-step $(\bullet)$ e three-step $(\bullet)$.

\section{DISCUSSÃO}

O objetivo deste estudo foi identificar a reprodutibilidade e a concordância de protocolos abreviados de marcha (one-step e three-step) direcionados à análise da PPD, utilizando como critério de comparação o protocolo padrão midgait. Os resultados encontrados apontaram aceitável reprodutibilidade para o PPP e a IPT; porém, dependendo da máscara podal considerada, com dimensões mais favoráveis ao protocolo three-step. Esses achados coincidem com estudo prévio que comparou a reprodutibilidade das medidas de PPD entre os protocolos abreviados one-step e three-step em pacientes com artrite crônica ${ }^{19}$. Nesse caso específico, também foi identificada reprodutibilidade de moderada a elevada magnitude em ambos os protocolos, 
com discreta vantagem para os coeficientes equivalentes ao protocolo one-step. Contudo, os investigadores responsáveis pelo estudo apontaram que o uso do protocolo three-step é mais oportuno, tendo em vista a similaridade nos indicadores de reprodutibilidade e o fato do protocolo one-step não produzir fase de apoio semelhante à marcha normal.

Em relação à concordância entre os protocolos, apesar dos protocolos abreviados demonstrarem tendência em subestimar as medidas de PPP e IPT produzidas pelo protocolo padrão midgait, na maioria das máscaras selecionadas não foram identificadas diferenças estatísticas entre os escores médios e os valores de $r$ se apresentaram significativos. Além disso, ao analisar informações adicionais por intermédio da técnica de Bland-Altman, constatou-se substancial capacidade de concordância nas medidas de PPP e IPT produzidas pelos protocolos abreviados e pelo protocolo padrão midgait. De maneira geral, esses achados são consistentes com estudos prévios ${ }^{13,14}$.

A princípio, considerando que as medidas de PPP e IPT são de fundamental importância para diagnóstico devidas as suas relações com processos álgicos, risco de ulceração, deformidades plantares e distúrbios musculoesqueléticos ${ }^{1,4,5}$ - e os protocolos abreviados se definem como mais accessíveis para uso clínico -, esses achados podem oferecer importantes implicações positivas. Entretanto, embora ambas as medidas de PPD sejam bastante úteis, investigadores e clínicos não devem realizar suposições ou inferências baseando-se exclusivamente na magnitude das medidas de pressão plantar. O PPP e a IPT demonstram ampla variação individual não sendo encontrado, até o momento na literatura, limiar previsto para a PPD que possa ser utilizado como referencial isolado de distúrbios da região plantar.

Por outro lado, os resultados do presente estudo podem estar em desacordo com os achados prévios ${ }^{20,21}$, que encontraram tendência para que as medidas obtidas mediante o uso do protocolo one-step se mostrem mais elevadas em comparação com os demais protocolos. No entanto, os investigadores chamam a atenção para o fato de que, embora seja possível identificar essa tendência, não foram apontadas diferenças significativas entre os escores. Justifica-se esse fenômeno com base no pressuposto de que existe relação direta entre aumento de velocidade e mais elevada medida de pressão plantar ${ }^{22}$. Dessa forma, em virtude do protocolo one-step partir de uma posição de repouso absoluto e do indivíduo entrar em contato com a plataforma na fase de aceleração da marcha, em tese, o resultado deverá apresentar medidas mais elevadas de PPD.
No estudo, entretanto, a velocidade da marcha não foi controlada, os participantes sendo orientados a caminhar em velocidade confortável e auto-selecionada. Associação entre velocidade de marcha e comportamento de PPD vem sendo abordada em diversos contextos e, até o momento, os achados têm demonstrado que cadência, ritmo, tipo de trajetória e inclinação do terreno desencadeiam diferentes comportamentos de $\mathrm{PPD}^{22,23}$. Desse modo, os protocolos abreviados fornecem diferentes demandas ao sistema neuromusculoesquelético, no sentido da interação do controle motor e da variabilidade biomecânica da tarefa desencadearem diferentes flutuações no centro de massa sobre a base de suporte e nos níveis de perturbação que desafiam a estabilidade e a coordenação ${ }^{22}$.

Para análise dos dados baropodométricos, optou-se, no presente estudo, por estratificar a estrutura podal em oito máscaras, considerando que essas áreas apresentam maior aplicação clínica nos casos de ulceração diabética, atividades esportivas e intervenções fisioterapêuticas. A divisão dos segmentos podais, porém, não é uniforme na literatura. Por exemplo, um estudo ${ }^{24}$ ao abordar a reprodutibilidade da distribuição de pressão plantar em indivíduos assintomáticos, estratificou o antepé em cinco máscaras, enquanto o presente estudo optou por realizar o mascaramento em três áreas podais. Ainda, os métodos utilizados para divisão segmentar do pé variam de acordo com o sistema de baropodometria utilizado e está sujeito a fragilidades devido à resolução do sistema, ao tipo de sensor da plataforma e ao grau de domínio da técnica ${ }^{25}$.

Nesse particular, Deschamps et al. ${ }^{26}$ realizaram experimento com o propósito de estabelecer a confiabilidade do mascaramento manual a partir da identificação da pressão plantar em $2 \mathrm{D}$ para análise do PPP e do tempo de contato em indivíduos com hálux valgo. Os resultados apontaram alta confiabilidade inter e intra-examinador, demonstrando padrão semelhante na definição das máscaras plantares, apesar de apresentar valores globais tendenciosamente menores quando tratados por mascaramento manual. Os investigadores concluíram que a realização de pequenas máscaras, como as que compreendem a região das cabeças dos metatarsos, deve ser analisada com cautela. Esse achado vem de encontro a outros estudos ${ }^{27,28}$ que demonstraram menor confiabilidade em mascaramentos de pequenas regiões podais, atribuindo esse efeito às limitações tecnológicas do equipamento, como tamanho e sensibilidade dos captores em isolar pequenas áreas plantares. Por esse motivo, no presente estudo, optou-se por realizar o mascaramento 
mediante definição automática fornecida pelo próprio software do equipamento.

Este estudo apresenta algumas limitações que devem ser consideradas quando da interpretação de seus resultados. No primeiro momento, os participantes recrutados e submetidos aos procedimentos eram jovens e saudáveis consequentemente, a validade externa dos achados torna-se prejudicada ao considerar sujeitos portadores de alguma disfunção ou de outras idades. Ademais, a ausência de controle de possíveis variáveis de confusão, como velocidade da marcha e nível de esforço físico feito previamente à coleta de dados, pode impactar sobre a reprodutibilidade da PPD. Além disso, as características da plataforma de pressão utilizada, como tamanho do captor, resolução espacial e pressão máxima por captor, podem afetar a sensibilidade e a acurácia dos dados de PPD, dificultando, por conseguinte, a comparação com investigações prévias que empregaram outros equipamentos.

\section{CONCLUSÃO}

Devido aos aceitáveis indicadores de reprodutibilidade e concordância observados nas diferentes máscaras podais, os protocolos abreviados de marcha podem ser empregados de forma satisfatória para identificar as medidas de distribuição de PPD. Apesar de não terem sido encontradas diferenças significativas, o protocolo one-step tendeu a subestimar os valores equivalentes ao PPP e à IPT, ao passo que o protocolo three-step apresentou mais adequada reprodutibilidade e dados mais próximos do protocolo padrão midgait. Portanto, na impossibilidade de uso do protocolo padrão midgait para identificar as medidas de PPD, sugere-se que seja empregado preferencialmente o protocolo abreviado threestep em jovens assintomáticos.

\section{REFERÊNCIAS}

1. Miura N, Nagai K, Tagomori K, Ikutomo H, Okamura K, Okuno $\mathrm{T}$, et al. Plantar pressure distribution during standing in women with end-stage hip osteoarthritis. Gait Posture. 2020;76:39-43. doi: 10.1016/j.gaitpost.2019.10.026

2. Wei Z, Zhang Z, Jiang J, Zhang Y, Wang L. Comparison of plantar loads among runners with different strike patterns. J Sports Sci. 2019;37(18):2152-8. doi: 10.1080/02640414. 2019.1623990

3. Hähni M, Hirschmüller A, Baur H. The effect of foot orthoses with forefoot cushioning or metatarsal pad on forefoot peak plantar pressure in running. J Foot Ankle Res. 2016;9:44. doi: 10.1186/s13047-016-0176-z
4. Chatwin KE, Abbott CA, Boulton AJM, Bowling FL, Reeves ND. The role of foot pressure measurement in the prediction and prevention of diabetic foot ulceration - A comprehensive review. Diabetes Metab Res Rev. 2020;36(4):e3258. doi: 10.1002/dmrr.3258

5. Hazari A, Maiya A, Agouris I, Monteiro A, Shivashankara. Prediction of peak plantar pressure for diabetic foot: The regressional model. Foot (Edinb). 2019;40:87-91. doi: 10.1016/j. foot.2019.06.001

6. Ma CZ, Zheng YP, Lee WC. Changes in gait and plantar foot loading upon using vibrotactile wearable biofeedback system in patients with stroke. Top Stroke Rehabil. 2018;25(1):20-7. doi: 10.1080/10749357.2017.1380339

7. Zulkifli SS, Loh WP. A state-of-the-art review of foot pressure. Foot Ankle Surg. 2020; 26(1):25-32. doi: 10.1016/j.fas.2018.12.005

8. Arts ML, Bus SA. Twelve steps per foot are recommended for valid and reliable in-shoe plantar pressure data in neuropathic diabetic patients wearing custom made footwear. Clin Biomech (Bristol, Avon). 2011;26(8):880-4. doi: 10.1016/j. clinbiomech.2011.05.001

9. Fascione JM, Crews RT, Wrobel JS. Association of footprint measurements with plantar kinetics: A linear regression model. J Am Podiatr Med Assoc. 2014;104(2):125-33. doi: 10.7547/0003-0538-104.2.125

10. Wearing SC, Urry S, Smeathers JE, Battistutta D. A comparison of gait initiation and termination methods for obtaining plantar foot pressures. Gait Posture. 1999;10(3):255-63. doi: 10.1016/ s0966-6362(99)00039-9

11. Bryant A, Singer K, Tinley P. Comparison of the reliability of plantar pressure measurements using the two-step and midgait methods of data collection. Foot Ankle Int. 1999;20(10):646-50. doi: 10.1177/107110079902001006

12. Bus SA, de Lange A. A comparison of the 1-step, 2-step, and 3-step protocols for obtaining barefoot plantar pressure data in the diabetic neuropathic foot. Clin Biomech (Bristol, Avon). 2005;20(9):892-9. doi: 10.1016/j.clinbiomech.2005.05.004

13. Izquierdo-Renau M, Pérez-Soriano P, Ribas-García V, Queralt A. Intra and intersession repeatability and reliability of the S-Plate ${ }^{\circledast}$ pressure platform. Gait Posture. 2017;52:224-6. doi: 10.1016/j.gaitpost.2016.12.001

14. Godi M, Turcato AM, Schieppati M, Nardone A. Test-retest reliability of an insole plantar pressure system to assess gait along linear and curved trajectories. J Neuroeng Rehabil. 2014;11:95. doi: 10.1186/1743-0003-11-95

15. Patrick K, Donovan L. Test-retest reliability of the Tekscan ${ }^{\circledast}$ F-Scan ${ }^{\circledast} 7$ in-shoe plantar pressure system during treadmill walking in healthy recreationally active individuals. Sports Biomech. 2018;17(1):83-97. doi: 10.1080/14763141.2017.1355010

16. Menz HB. Analysis of paired data in physical therapy research: time to stop double-dipping? J Orthop Sports Phys Ther. 2005;35(8):477-8. doi: 10.2519/jospt.2005.0108

17. Nouman M, Dissaneewate T, Leelasamran W, Chatpun S. The insole materials influence the plantar pressure distributions in diabetic foot with neuropathy during different walking activities. Gait Posture. 2019;74:154-61. doi: 10.1016/j.gaitpost.2019.08.023

18. Borg J, Mizzi S, Formosa C. Peak pressure data and pressuretime integral in the contralateral limb in patients with diabetes and a trans-tibial prosthesis. Gait Posture. 2018;64:55-8. doi: 10.1016/j.gaitpost.2018.05.023. 
19. Van der Leeden M, Dekker JH, Siemonsma PC, Lek-Westerhof SS, Steultjens MPM. Reproducibility of plantar pressure measurements in patients with chronic arthritis: a comparison of one-step, two-step, and three-step protocols and an estimate of the number of measurements required. Foot Ankle Int. 2004;25(10):739-44. doi: 10.1177/107110070402501008

20. Peters EJG, Urukalo A, Fleishli JG, Lavery LA. Reproducibility of gait analysis variables: one-step versus three-step method of data acquisition. J Foot Ankle Surg. 2002;41(4):206-12. doi: 10.1016/s1067-2516(02)80016-3

21. Harrison AJ, Folland JP. Investigation of gait protocols for plantar pressure measurement of non-pathological subjects using a dynamic pedobarograph. Gait Posture. 1997;6(1):50-5. doi: 10.1016/S0966-6362(96)01095-8

22. McClymont J, Pataky TC, Crompton RH, Savage R, Bates KT. The nature of functional variability in plantar pressure during a range of controlled walking speeds. R Soc Open Sci. 2016;3(8): 1-14. doi: 10.1098/rsos.160369

23. Fan Y, Li Z, Han S, Lv C, Zhang B. The influence of gait speed on the stability of walking among the elderly. Gait Posture. 2016;47:31-6. doi: 10.1016/j.gaitpost.2016.02.018
24. Xu C, Wen XX, Huang LY, Shang L, Cheng XX, Yan YB, Lei $W$. Normal foot loading parameters and repeatability of the Footscan ${ }^{\oplus}$ platform system. J Foot Ankle Res. 2017;10:30. doi: 10.1186/s13047-017-0209-2

25. Telfer S, Bigham JJ. The influence of population characteristics and measurement system on barefoot plantar pressures: A systematic review and meta-regression analysis. Gait Posture. 2019;67:269-76. doi: 10.1016/j.gaitpost.2018.10.030

26. Deschamps K, Birch I, McInnes J, Desloovere K, Matricali GA. Inter- and intra-observer reliability of masking in plantar pressure analysis. Gait Posture. 2009;30(3):379-82. doi: 10.1016/j.gaitpost.2009.06.015

27. Wallace J, White H, Augsburger S, Walker J. Development of a method to produce a valid and reliable foot mask for plantar pressure evaluation in children with clubfoot. J Pediatr Orthop B. 2020 [Preprint]. doi: 10.1097/BPB.0000000000000801

28. Giacomozzi C, Stebbins JA. Anatomical masking of pressure footprints based on the Oxford Foot Model: validation and clinical relevance. Gait Posture. 2017;53:131-8. doi: 10.1016/j. gaitpost.2016.12.022 